\title{
The newly improved set-up at the Planetary Spectroscopy Laboratory (PSL)
}

\author{
A. Maturilli*a, J. Helbert ${ }^{\mathrm{a}}$, G. Arnold ${ }^{\mathrm{a}}$ \\ ${ }^{a}$ Institute of Planetary Research, German Aerospace Centre (DLR), 2 Rutherfordstrasse, Berlin, \\ Germany 12489 \\ *alessandro.maturilli@dlr.de; phone +49 030 67055313; $\underline{\text { dlr.de/pf/ }}$
}

\begin{abstract}
The Planetary Spectroscopy Laboratory (PSL) of DLR in Berlin provides spectral measurements of primarily planetary analogues from the visible to the far-infrared range. PSL has supported the data analysis as well as the development and calibration of instruments for planetary missions from ESA, NASA and JAXA. For this purposes PSL provides reflection, transmission and emission spectroscopy of target materials.
\end{abstract}

Currently PSL operates three identical Bruker Vertex 80V vacuum FTIR spectrometer (the third one just installed in June 2019), two spectrometers are equipped with aluminum mirrors optimized for the UV, visible and near-IR, the third features gold-coated mirrors for the near to far IR spectral range.

External simulation chambers are attached to two of the instruments for emissivity measurements. The chamber at the near to far IR instrument allows emissivity measurements from 0.7-200 $\mu \mathrm{m}$ under vacuum for sample temperatures from $320 \mathrm{~K}$ to above $900 \mathrm{~K}$, using an innovative induction system. The second chamber (purged with dry air and water cooled to $\leq 270 \mathrm{~K}$ ) allows emissivity measurements of samples with surface temperature from $290 \mathrm{~K}$ to $420 \mathrm{~K}$.

We measure bi-directional reflectance of samples; with variable incidence and emission angles between $0^{\circ}$ and $85^{\circ}$ (minimum phase angle is $26^{\circ}$ to prevent damages to the mirrors). Samples are measured currently at room temperature and $170 \mathrm{~K}$, with a planned extension for temperatures below $100 \mathrm{~K}$, by means of a new external chamber, whose funding is accepted and will be available in 2020 .

Bi-directional and hemispherical reflectance is measured under purging/vacuum conditions, covering the 0.2 to above $200 \mu \mathrm{m}$ spectral range.

An FT-IR microscope installed at the end of 2018, allows microscopic analysis in transmission and reflectance in the VIS+VNIR+MIR spectral range.

Transmission of thin slabs, optical filters, optical windows, pellets, and others is measured in the complete spectral range from UV to FIR using a parallel beam configuration to avoid refraction.

Keywords: Planetary Analogues, Spectroscopy of Minerals, Mineralogy, Infrared, Mercury, Venus, Moon, Asteroid

\section{INTRODUCTION}

Spectroscopy is still the most accurate methodology to remotely study the surface composition of celestial bodies (and its evolution). For more than fifteen years the Planetary Emissivity Laboratory (PEL) of DLR in Berlin has provided spectral measurements of planetary analogues from the visible to the far-infrared range for comparison with remote sensing spacecraft/telescopic measurements of extra-terrestrial surfaces [1-5]. Reflection, transmission and emission spectroscopy are the techniques we used to acquire spectral data of target materials.

A recent major upgrade to our laboratory set-up added a new spectrometer, a FT-IR microscope, three external sources, optical units, new detectors (among them, a new MCT wide band detector) and beamsplitters to further extend the spectral range of measurements that can be performed in the laboratory, as well as the temperature range that we can cover for the measurements. The FT-IR microscope was successfully installed at the end of 2018, allowing for reflectance and transmission microscopy. The purpose of this paper is to illustrate the very wide range of capabilities that the Planetary Spectroscopy Laboratory (PSL) can offer to the planetary and to the spectroscopic community. 


\section{PSL SET-UP DESCRIPTION}

Three identical FTIR (Fourier Transform Infrared Spectrometers) instruments are operated at PSL, in an air-conditioned laboratory room (Figure 1). The 3 spectrometers are all the same identical model, Bruker Vertex $80 \mathrm{~V}$ that can be evacuated to .1 mbar. Two spectrometers are equipped with aluminum mirrors optimized for the UV, visible and nearIR, the third features gold-coated mirrors for the near to far IR spectral range. Using three instruments that are identical (apart from the different internal mirrors) has some major benefits. Most importantly it facilitates the cross-calibration between the three instruments. The instruments can also share the collection of detectors, beamsplitters, and optical accessories that are available in our equipment to cover a very wide spectral range. The instruments and the optical accessory units used are fully automatized and the data calibration and reduction are performed with software developed following the DLR quality management rules. Table 1 list the collection of detectors that we have available at PSL and the spectral range in which those devices are used, Table 2 describes the associated beamsplitters we have in use at PSL.

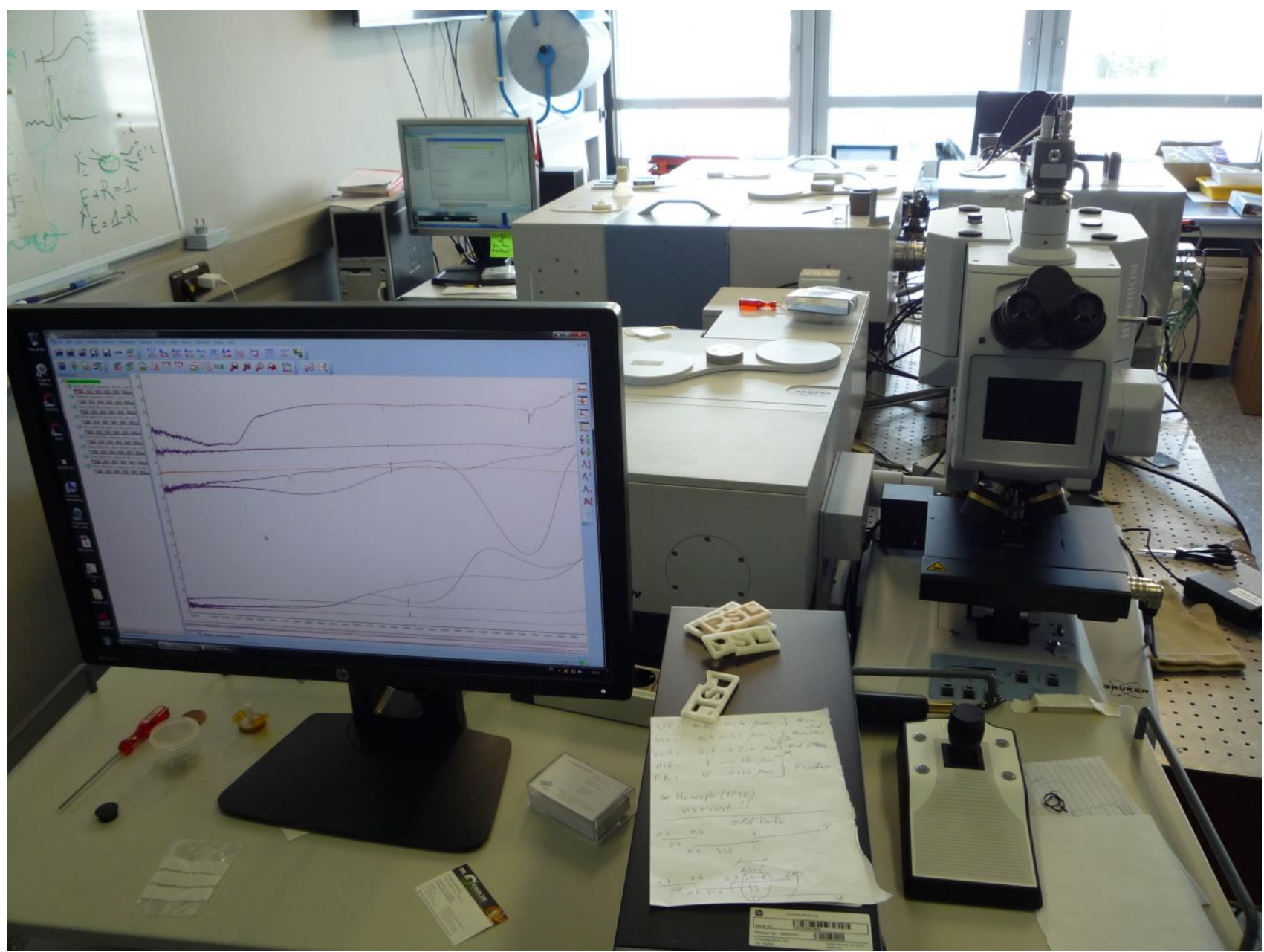

Figure 1. The Laboratory set-up at PSL with view of the 3 spectrometers and the microscope visible in foreground. 
Table 1. Collection of detectors equipment available at PSL.

\begin{tabular}{|l|l|l|}
\hline \multicolumn{1}{|c|}{ Detector } & \multicolumn{1}{c|}{ Spectral Range $(\boldsymbol{\mu m})$} & \multicolumn{1}{c|}{ Operating T } \\
\hline GaP Diode & $0.2-0.55$ & Room T \\
\hline 2x Silicon Diode & $0.4-1.1$ & Room T \\
\hline 2x InGaAs Diode & $0.7-1.8$ & Room T \\
\hline InSb & $0.78-5.4$ & Liquid $\mathrm{N}_{2}$ \\
\hline 2x MCT & $0.8-16$ & Liquid $\mathrm{N}_{2}$ \\
\hline MCT wide band & $0.8-25$ & Liquid $\mathrm{N}_{2}$ \\
\hline MCT/InSb SW & $1-16$ & Liquid $\mathrm{N}_{2}$ \\
\hline 3x DTGS/KBr & $0.8-40$ & Room T \\
\hline DTGS/CsI & $0.8-55$ & Room T \\
\hline DTGS/PE & $14-1000$ & Room T \\
\hline
\end{tabular}

Table 2. Collection of beamsplitters equipment available at PSL.

\begin{tabular}{|l|l|}
\hline \multicolumn{1}{|c|}{ Beamsplitter } & \multicolumn{1}{c|}{ Spectral Range $(\boldsymbol{\mu m})$} \\
\hline 2x UV/VIS/NIR $\mathrm{CaF}_{2}$ & $0.18-2.5$ \\
\hline 2x Si on $\mathrm{CaF}_{2}$ & $0.66-8.3$ \\
\hline 3x Ge on $\mathrm{KBr}($ Wide $)$ & $1-25$ \\
\hline Ge on $\mathrm{KBr}$ substrate & $1.2-25$ \\
\hline Multilayer & $14.7-333$ \\
\hline $50 \mu \mathrm{m} \mathrm{Mylar}$ & $181-666$ \\
\hline 2x DTGS/KBr & $0.8-40$ \\
\hline
\end{tabular}

Three external sources feature the PSL set-up: a deuterium lamp is used to cover the UV (0.2 to $0.5 \mu \mathrm{m})$ spectral range; a $24 \mathrm{~V}$, water cooled, Tungsten lamp has been added for measurements in the VIS (0.4 to $1.1 \mu \mathrm{m})$ spectral range; high power Globar lamp ( $24 \mathrm{~V}$, water cooled) is used in the VNIR+TIR (1 to $16 \mu \mathrm{m})$ spectral range. Complimentary to the external ones, two internal sources from VIS to FIR are installed in each spectrometer to complete the available offer.

\section{PSL SUPPORT EQUIPMENT}

Sample preparation, analysis tools, and experiment sub-systems are available at the facility. The full layout is shown in Figure 2. We have a collection of hundreds of rocks and minerals, several synthetic minerals, one Apollo 16 lunar sample, and many meteorites (bulk or powder form). This is complemented by our sample preparation and characterization lab operated by a dedicated technician. This includes two milling setups, two automated sieving setups with a wide range of sets for sieving, an ultrasonic cleaning unit, grinders, mortars, saw, balances, two stereo microscopes, two ovens ( 290 to $570 \mathrm{~K}$ and 300 to $3300 \mathrm{~K}$ ) for sample treatment, wet chemistry materials, and a pellets press $(10 \mathrm{~mm}$ or $20 \mathrm{~mm}$ diameter). Typical grain size separates produced for our spectral measurements are $<25 \mu \mathrm{m}$, 25 $63 \mu \mathrm{m}, 63-125 \mu \mathrm{m}, 125-250 \mu \mathrm{m}$; larger separates as well as slabs are produced anytime is needed. For storing sensitive samples we have three large dry cabinets (moisture < 1\%) for sample storage as well as several small desiccators (moisture $<20 \%$ ). A purge gas generator for water and $\mathrm{CO}_{2}$ free air and a liquid-nitrogen tank complements the technical lab support. 


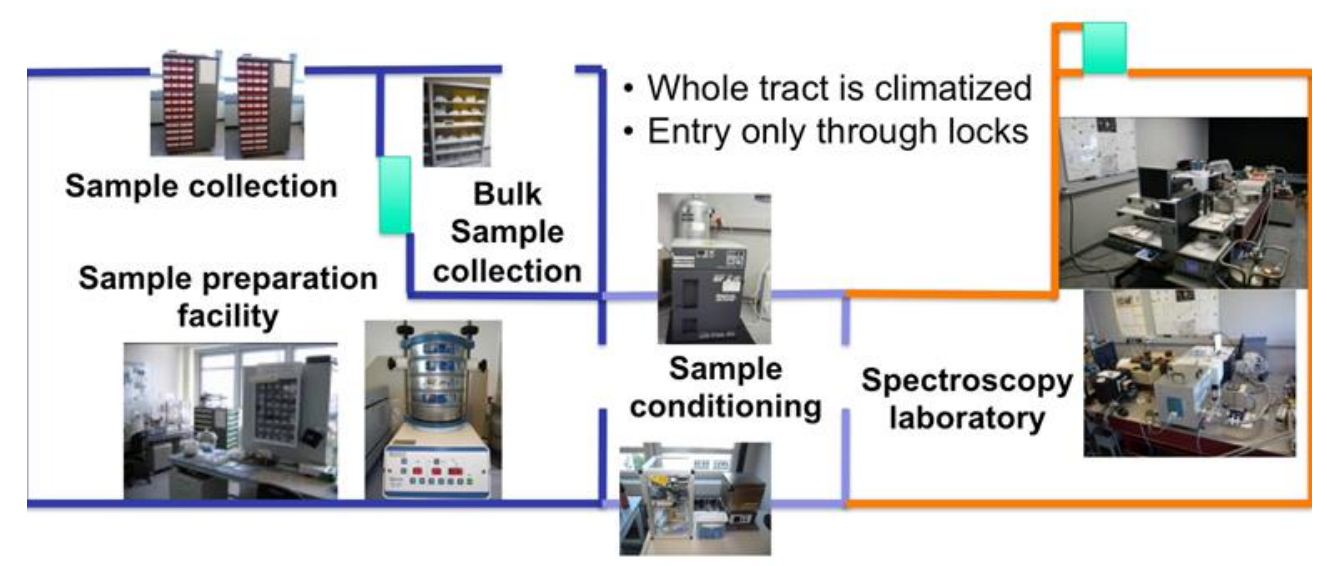

Figure 2. Sketch of the support equipment at the PSL.

Toward the end of 2018 a new Bruker HYPERION 2000 FT-IR Microscope was installed at PSL to be operated together with one of the Vertex $80 \mathrm{~V}$ spectrometers. With this device, samples can be very precisely analyzed in transmittance and in reflection. The spectral range of the HYPERION can be extended from the middle infrared to the near infrared (NIR), even to the visible (VIS, down to $0.4 \mu \mathrm{m}$ ) and up to the far infrared (FIR, up to $1000 \mu \mathrm{m}$ ). To cover this extremely broad spectral range, many different detectors are available and can easily be exchanged by the user. The HYPERION can be equipped with up to two detectors in parallel, where the switching between positions is controlled by the software delivered together with the instrument. The IR Microscope features a Linkam FTIR600 sample stage, to allow infrared analysis of samples from -196 up to $600^{\circ} \mathrm{C}$ (with both fast heating and cooling of the sample). Different window materials such as Zinc Selenide and Barium Fluoride are available to cover a wide spectral range of measurements.

Building on the available infrastructure and on the long heritage collected in the last almost 15 years of PEL/PSL, DLR is planning to create a Planetary Sample Analysis laboratory (PSA), which can be later extended to a full Sample Curation facility. PSA will focus on spectroscopy on the microscopic scale and geochemical analysis methods to study elemental composition and isotopic ratios in addition to mineralogy to derive information on the formation and evolution of planetary surface search for traces of organic materials and inclusions of water. To characterize and analyze the samples (including extra-terrestrial returned samples) we will add an Electron Microprobe Analyzer (EMPA) for elemental analysis; a Laser ablated inductive coupled Plasma Mass Spectrometer for elemental and isotope analysis, and a Dual Source TXRF \& Grazing Incidence ED-XRF for mineralogical and structural analysis. The DLR PSA will be operated as a community facility (much like PSL), supporting the larger German and European sample analysis community.

\section{SPECTRAL MEASUREMENT OF SAMPLE EMISSIVITY}

External simulation chambers are attached to two of the FTIR spectrometers to measure the emissivity of solid samples. One chamber features a high efficiency induction system to heat samples under vacuum to temperatures from $320 \mathrm{~K}$ up to above $900 \mathrm{~K}$, while keeping the chamber at almost ambient temperature. A shutter allows separating the spectrometer from the external chamber. The advantages of a shutter separating the external chamber from the spectrometer are plentiful: samples can be heated at vacuum pressure lower than that available inside the spectrometer (vacuum in the spectrometer is limited by the air-bearing necessary for the mirror movements); in case of outgassing, no harm is caused to the sensible optical parts inside the spectrometer; optical parts inside the spectrometer are separated from the hot sample environment, hence they are not heated, also their optical properties are not changing during a day of measurements; spectrometer can be vented and optical units exchanged while the samples in the external chamber remains in vacuum, allowing simultaneous measurements on a wider spectral range. An optical window (vacuum tight) can be mounted at the entrance of the emissivity chamber to allow keeping the external chamber at $\geq$ ambient pressure, 
under purged air or inert gases. The sample cups are made of stainless steel and have elevated rims enclosing the samples heating it from all the sides, effectively suppressing thermal gradients within the samples. Aluminum sample cups are available for samples that need to be heated at lower temperatures. A sample carousel driven by a highly precise stepper motor allows measuring several consecutive samples without breaking the vacuum. A large number of temperature sensors in the emissivity chamber are allocated to measure the sample/cup temperature as well as monitoring the equipment and chamber temperatures. A webcam is mounted in the emissivity chamber to monitor the heated sample and its vicinity; it is usually used to take pictures of the sample at each measuring temperature, but can also be used to obtain a video-recording of an experiment. The latter can add precious information on the thermal processes occurring to the heated materials in the emissivity chamber. The flexible design of the camber allows adding other monitoring devices in the chamber (e.g. IR camera, gas fugacity sensor, and others).

Each sample to be measured is poured in a stainless steel / aluminum cup to a $3 \mathrm{~mm}$ thick uniform layer; the cup is placed on an induction pancake-coil. Induction heating is used to heat up the cup and thereby the samples contained. By using the induction properties to heat the cups, we do not set the heater temperature, but the intensity of the current that we send to the induction coil. The whole cup (made of steel or aluminum, that heats up weaker than steel) is heated uniformly by the induction process; therefore our samples are heated from below as well as from the side (rims) of the cup, and are not heated/illuminated from above. At least one thermopile temperature sensor (whose head is a long, thin wire of $0.2 \mathrm{~mm}$ diameter) is put in contact with the emitting surface, to read the sample temperature. Blast furnace slag, poured in a sample cup and under exactly the same geometric configuration as the samples, is heated to sample temperature and is used as calibration blackbody. The calibrated sample emissivity is then calculated by dividing the sample radiance to the blackbody radiance (measured at the same temperature), and then multiplying this resulting spectrum for the emissivity spectrum of the blackbody material. The emissivity curve of the slag has been retrieved by measuring the blast furnace slag vs. a commercial blackbody, painted with a black and well characterized coating (Acktar Fractal Black). Figure 3 shows an example of emissivity measurement taken in vacuum for a quartz sample (125-250 $\mu \mathrm{m}$ grain size range) at various high temperatures. It can be noted that for the emissivity spectrum for this sample taken at $666^{\circ} \mathrm{C}$ surface temperature shows the spectral effects of the $\alpha$ - to $\beta$ - transition typical for the tetrahedral molecule of quartz (a continuous framework of $\mathrm{SiO}_{4}$ ). In fact, the room-temperature form of quartz, $\alpha$-quartz, undergoes a reversible change in the crystal structure at $573{ }^{\circ} \mathrm{C}$ to form the $\beta$-quartz.

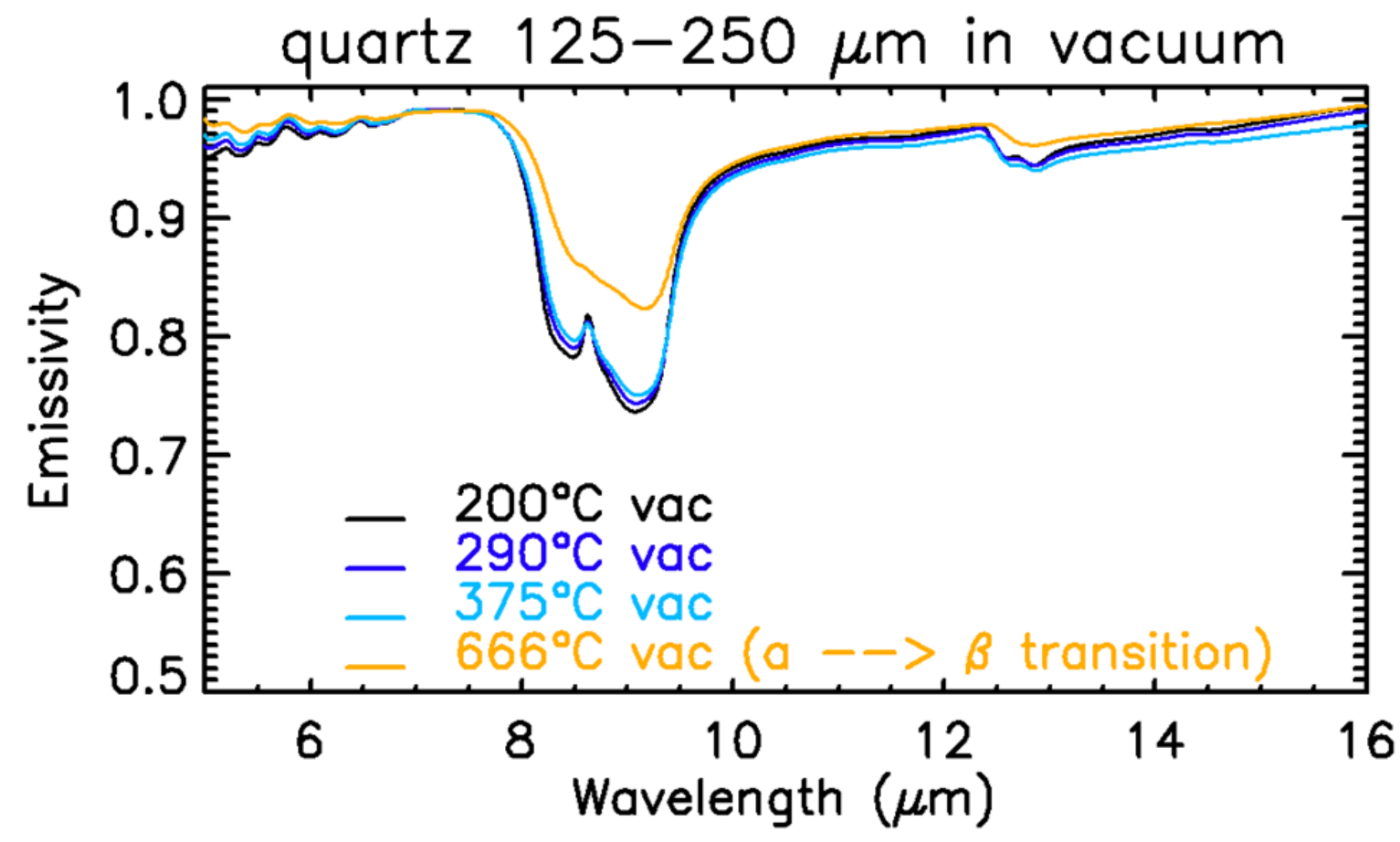

Figure 3. Emissivity spectra of a quartz sample taken in vacuum at increasing temperatures. 
In a recent upgrade we added capabilities to measure emissivity at wavelengths as low as $0.7 \mu \mathrm{m}$, to specifically address the needs of the Venus science community. This upgrade was funded by the European Commission European Union's Horizon 2020 research and innovation program under grant agreement No 654208. The permanent cloud cover of Venus prohibits observation of the surface with traditional imaging techniques over most of the visible spectral range. Venus' $\mathrm{CO}_{2}$ atmosphere is only transparent in small spectral windows near $1 \mu \mathrm{m}$. However, using stainless steel cups to heat samples to very high temperatures has a disadvantage in the visible spectral range. As the emissivity of steel increases significantly in the $1 \mu \mathrm{m}$ region at this high temperature the steel cup is visibly glowing and the emitted radiation from the sample cup can exceed the sample emitted energy measured in the visible spectral range. To bypass this difficulty, ceramic cups enclosing a stainless steel disc are used to extend the high temperature spectroscopy capabilities of PSL to start at 700nm instead of 1000nm (see Figure 4).

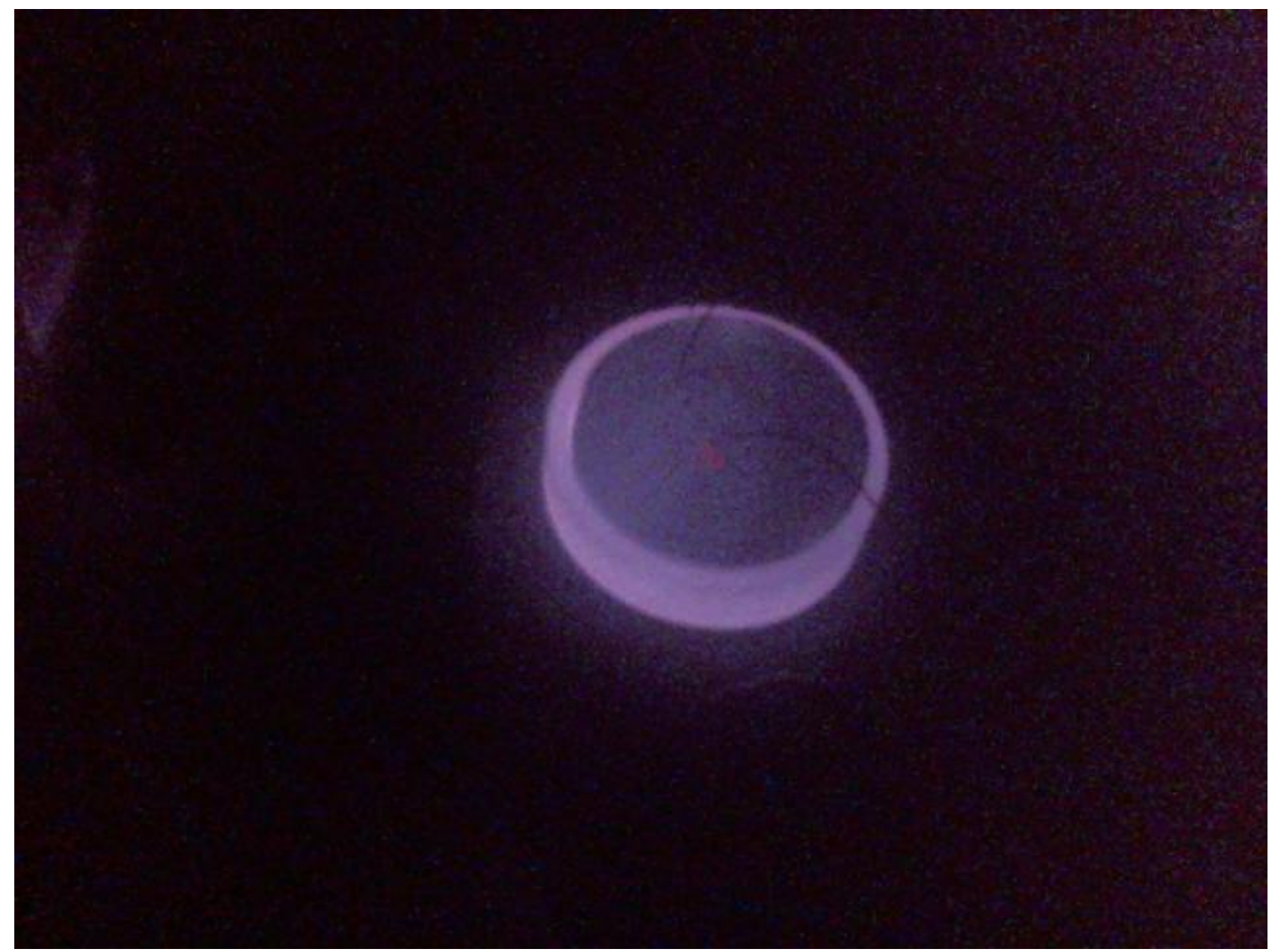

Figure 4. Webcam picture (taken with lights off) of a sample cup, sample measured surface temperature is $480^{\circ} \mathrm{C}$.

A second chamber (purged with dry air and water cooled to $280 \mathrm{~K}$ or below) is attached to the second spectrometer for emissivity measurements of samples with surface temperature from 290 to 420K. This second emissivity chamber is well suited for emissivity measurements of samples at temperatures close to room. It is covered with high emissivity black paint, also its walls reflect almost zero signals, while at the same time the cooled black walls emit very low signal.

Standard spectral resolution used for emissivity measurements is $4 \mathrm{~cm}-1$ and the spot size is 49 mm for both emissivity set-ups. We have been working to reduce the spot size, hence the amount of material needed to fill one emissivity cup. Coupling a reduced size mirror with reduced size sample cups, we succeeded to produce emissivity measurements with 
very good signal to noise, but on a limited amount of sample, reaching the goal of $<2$ grams of sample needed for each cup.

\section{SPECTRAL MEASUREMENT OF SAMPLE REFLECTANCE}

With the Bruker A513 accessory on both Vertex 80V spectrometers, we measure bi-directional reflectance of samples, with variable incidence and emission angles between $0^{\circ}$ and $85^{\circ}$ (minimum phase angle is $26^{\circ}$ ). The viewing cone of the A513 reflectance accessory has an aperture of $17^{\circ}$, small enough to define our measurements as bi-directional. Samples can be measured at room temperature and currently to $170 \mathrm{~K}$ using a test setup cooled by liquid nitrogen inside the spectrometer sample chamber. A compact low-temperature reflectance chamber for FT-spectroscopy experiments at the PSL is currently under development [7]. The expected cryogenic temperature to reach is approximately within the range of $70 \mathrm{~K}-100 \mathrm{~K}$. We recently added two integrating spheres (one with gold coated surfaces, the other with PTFE coating) for hemispherical reflectance measurements under purging air or vacuum, after a modification of the sample compartment hardware wall for the Vertex $80 \mathrm{~V}$ spectrometer. Reflectance measurements are calibrated by comparing with spectroscopic measurements of well characterized references. Standard spectral resolution used is $4 \mathrm{~cm}^{-1}$ and spot size is $2 \mathrm{~mm}$ for reflectance acquisitions. We measure bi-directional and hemispherical reflectance under purging or vacuum conditions, covering the 0.2 to above $200 \mu \mathrm{m}$ spectral range. Figure 5 shows an example of bi-directional reflectance measurement for several phase angles that we can perform on a very wide spectral range with our instruments at PSL. The sample measured is a synthetic MnS sulfide, and it was used because it is a suggested sulfide material that can be found on the surface of Mercury.
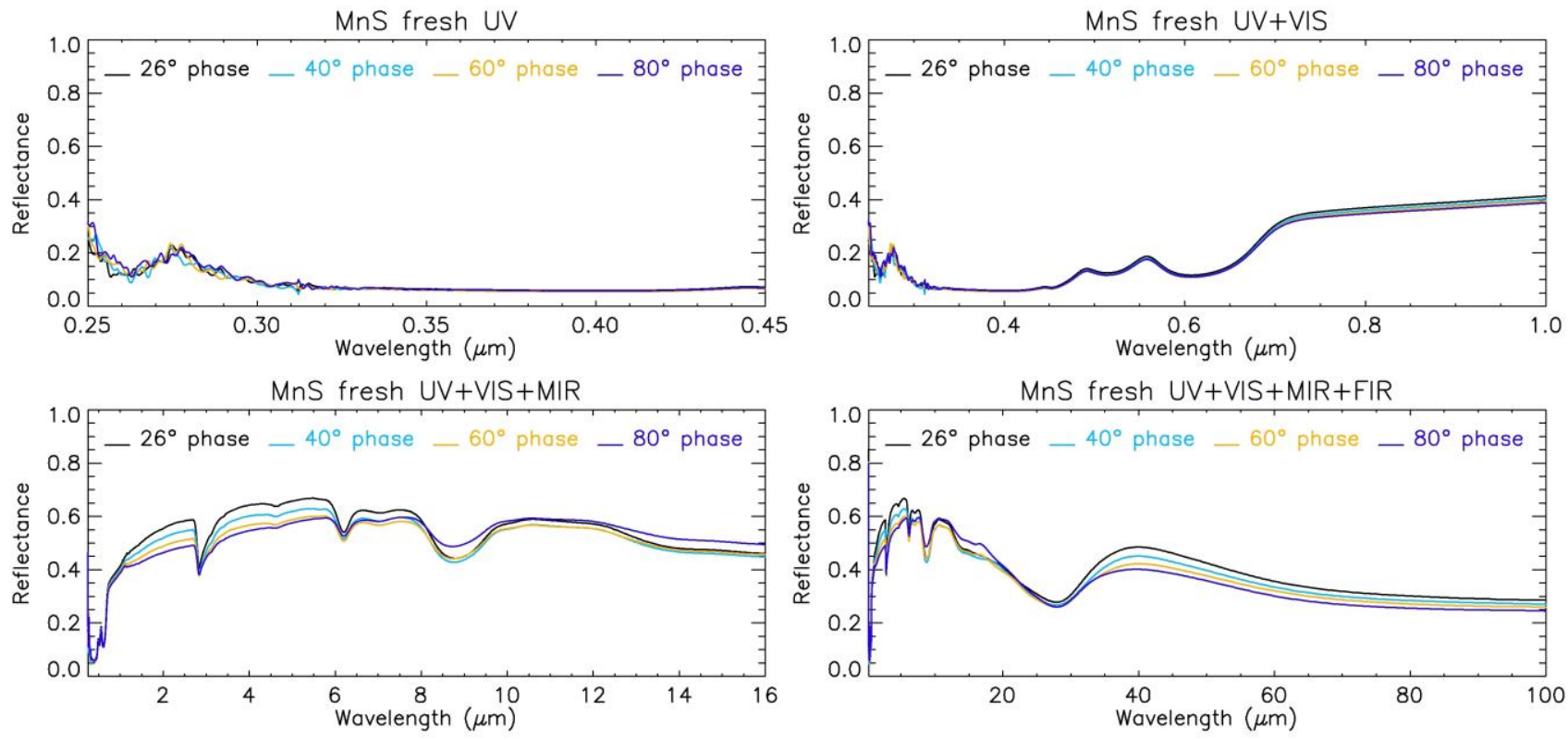

Figure 5. Bi-directional reflectance spectrum of the MnS sample measured at the PSL in the whole spectral range from UV to the FIR $(0.2-150 \mu \mathrm{m})$ and with several phase angles.

\section{SPECTRAL MEASUREMENT OF SAMPLE TRANSMITTANCE}

The Bruker A480 parallel beam accessory mounted on the Vertex $80 \mathrm{~V}$ allows us to measure transmission of thin slabs, optical filters, optical windows, pellets, etc., in the complete spectral range from UV to FIR avoiding refraction, typical in this kind of measurements. Especially in the case of thick and highly refractive samples, refraction causes a shift of the IR-focus towards the detector, which is associated with an error in the transmittance values.

For oblique incidence, refraction gives rise to an additional error caused by a lateral beam shift. These inaccuracies can be overcome by measuring the sample in a parallel IR-beam with perpendicular incidence, as is realized in the parallel 
beam transmission accessory A480. Figure 6 shows the comparison between a sample measured in transmittance with the equipment available at PSL and a transmittance spectrum of the same specimen taken from [8]. Our sample for transmittance measurements was prepared by mixing together finely powdered $99 \% \mathrm{KBr}$ (in weight) and $1 \%$ quartz powder. The mixture was then pressed in vacuum with a Graseby Specac Hydraulic Press at 10 ton for 10 minutes. The transmittance spectrum of obtained pellet could then be measured in vacuum in one of our Bruker Vertex 80V spectrometer. Transmittance measurements become a valuable additional opportunity at PSL for several reasons. It can be a very valuable analysis method in case of thin sections, for example of meteorites. Furthermore, our institute is constantly developing new flight instruments for planetary missions: thanks to the PSL transmission equipment we can characterize filters, optical windows and other optical elements building the flight instrument.

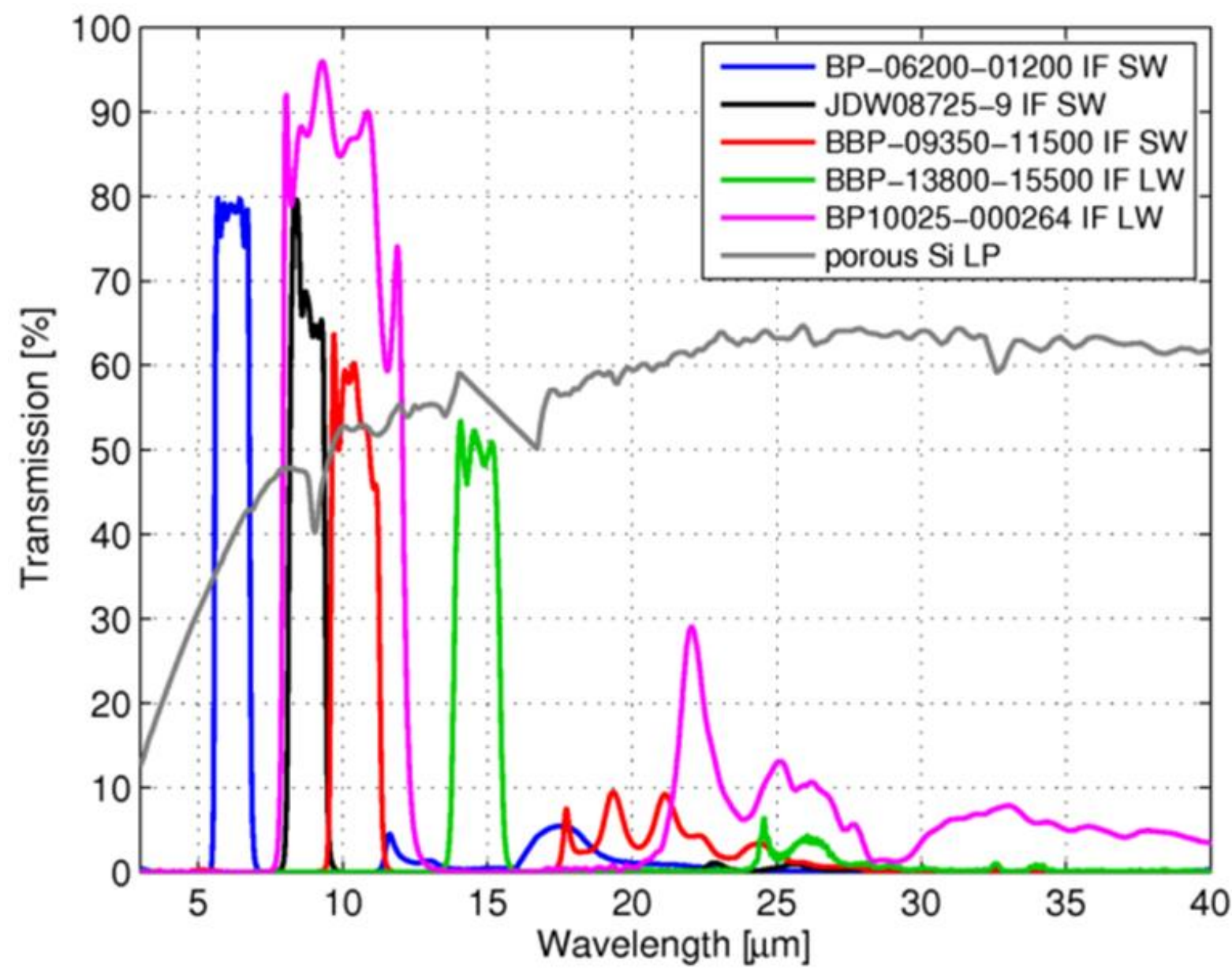

Figure 6. Transmittance spectrum of the 4 bandpass filters (measurements performed at PSL) for the instrument MARA on the Hayabusa2 lander MASCOT.

\section{FT-IR MICROSCOPIC SPECTRAL MEASUREMENTS}

The Bruker HYPERION 2000 FT-IR microscope installed at PSL provides high stability and reliability, for reflectance and transmission microscopy. To investigate samples at certain temperatures from -196 to $600^{\circ} \mathrm{C}$, the Linkam THM600 stage is used. ATR and Grazing Angle Objective (GAO) are available for sample viewing without sacrificing infrared light throughput and for measurement of very thin coatings. The HYPERION can be equipped with up to two detectors in parallel, where the switching between positions is controlled by the same software controlling the spectrometers. The HYPERION packs diverse techniques to enhance the visual quality of the sample in transmission or reflection. 
To select the most appropriate visualization for a certain sample, the HYPERION is equipped with a nosepiece and a wide variety of objectives. Rotatable polarizers are available in transmission and reflection that enable the user to distinguish samples exhibiting birefringence. For samples that mostly scatter light, dark field illumination can be applied. An autofocus function simplifies the inspection of structured sample surfaces.

The Bruker's all-in-one IR spectroscopy software OPUS provides numerous tools to ideally analyze the sample visually. One can measure distances and objects with a click of the mouse or add annotations and zoom in to interesting areas. All visual images are saved alongside the measured infrared spectra and their specific sampling positions. Binoculars are available to provide a sample view with highest optical quality. The CCD image of the sample is not only shown within the OPUS software but also on an optional screen that is integrated into the microscope housing.

Figure 7 shows an example of FT-IT Microscope image and spectral classification for the thin section of a weathered meteorite (enstatite chondrite), where spectral differences associated with different degree of weathering can be noticed.
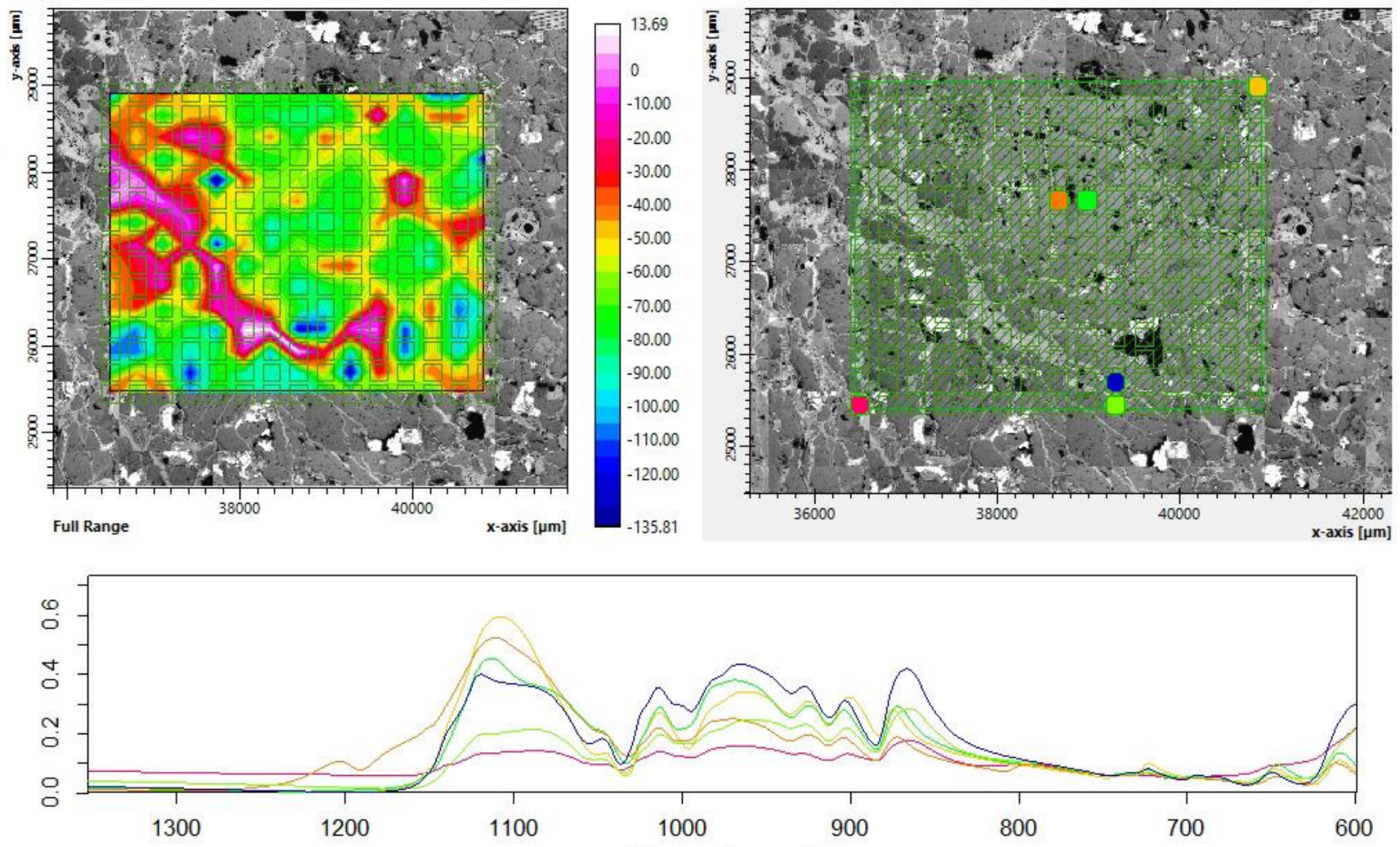

Wavenumber $\mathrm{cm}-1$
Figure 7. 2D mid-IR (1300-650 $\mathrm{cm}^{-1}$ ) reflectance map of a thin section for a space-weathered meteorite (an enstatite chondrite). Top left: contour map of the reflectance measurement points: top right: each square is the correspondent measurement point, the area of each point correspond to the detector area. Bottom: reflectance spectra of the highlighted points in the map above. The orthoenstatite spectra clearly show different band shapes according to the degree of space weathering they underwent.

\section{CONCLUSION}

The Planetary Spectroscopy Laboratory (PSL) is constantly improving to provide the planetary and spectroscopic community with reflectance, transmission and emissivity measurements highly complementary to existing spectral databases. PSL offer the unique opportunity to use 3 units of the same spectrometer type to perform spectroscopy under vacuum (or purged air), covering the whole spectral range from UV $(0.2 \mu \mathrm{m})$ to the FIR (200 $\mu \mathrm{m}$ and above), and for sample temperature from $70 \mathrm{~K}$ to $1000 \mathrm{~K}$.

A new IR Microscope is installed in PSL since the end of 2018 to provide a new powerful tool for small and sensitive sample analysis and spectroscopy on samples from $70 \mathrm{~K}$ to $900 \mathrm{~K}$.

A large collection of minerals (natural and synthetic), rocks, meteorites, and an Apollo 16 lunar sample are part of our sample collection. Complimentary sample preparation and characterization laboratory is equipped with plenty of devices 
for crushing, milling, and sieving the samples. Two ovens ( 290 to $570 \mathrm{~K}$ and 300 to $3300 \mathrm{~K}$ ) allow for sample treatment, three large dry cabinets and few small desiccators are used to store sensitive samples.

Future plans foreseen the creation of a Sample Analysis Laboratory (SAL) featuring an Electron Microprobe Analyzer (EMPA) for elemental analysis; a Laser ablated inductive coupled Plasma Mass Spectrometer for elemental and isotope analysis, and a Dual Source TXRF \& Grazing Incidence ED-XRF for mineralogical and structural analysis.

PSL is a Trans-national access (TA) facility supported by the European Union within the Europlanet Research Infrastructure framework for the next two years. Within this period once per year a call for proposals will be issued for investigations using PSL.

PSL is also member of the NASA SSERVI Team TREX [9] providing spectral measurements of fine particle samples (https://trex.psi.edu/).

\section{REFERENCES}

[1] Maturilli, A. and Helbert, J, "Emissivity measurements of analogue materials for the interpretation of data from PFS on Mars Express and MERTIS on Bepi-Colombo", Planetary and Space Science 54, 1057-1064 (2006).

[2] Maturilli, A., Helbert, J., and Moroz L., "The Berlin Emissivity Database (BED)", Planetary and Space Science 56, 420-425 (2008); spectral library now available at http://figshare.com/articles/BED_Emissivity_Spectral_Library/1536469.

[3] Helbert, J. and Maturilli, A, "The emissivity of a fine-grained labradorite sample at typical Mercury dayside temperatures", Earth and Planetary Science Letters 285(3), 347-354 (2009).

[4] Maturilli A, Helbert J., "Characterization, testing, calibration, and validation of the Berlin emissivity database", Journal of Applied Remote Sensing (2014).

[5] Maturilli A, Helbert J, St. John J.M., Head III J.W., Vaughan W.M., D’Amore M., Gottschalk M., Ferrari S., "Komatiites as Mercury Surface Analogues: Spectral Measurements at PEL", Earth and Planetary Science Letters 398, 58-65 (2014).

[6] Helbert, J., Maturilli, A., Dyar, M. D., Ferrari, S., Müller, N., Smrekar, S., "Orbital Spectroscopy of the Surface of Venus", Lunar and Planetary Science Conference, Abstract\# 1219 (2018).

[7] Rosas Ortiz, Y. M., Helbert, J., Maturilli, A., Lehman, M., "A compact Planetary Simulation Chamber for the Characterization of the Bi-directional Reflectance of Asteroid, Cometary and Solar System Small Bodies (SSSB) analogues at Low-Temperature Environments", Lunar and Planetary Science Conference, Abstract\# 1883 (2018).

[8] Farmer, V. C., "The Infrared Spectra of Minerals", Mineralogical Society Monograph 4, 1-539 (1974).

[9] Lane, M. D., Allain, J. P., Cahill, K. S., Clark, R. N., Cloutis, E. A., Dyar, M. D., Helbert, J., Hendrix, A. R., Holsclaw, G., Osterloo, M., Pearson, N., Savin, D. W., and the TREX team, "Toolbox for Research and Exploration (TREX): The Fine-Particle Spectral Library”, Lunar and Planetary Science Conference, Abstract\# 1098 (2018). 